\title{
Is there an association between adolescents' high caloric food portion size and body mass index?
}

\author{
S. A. Albar, N. A. Alwan, C. E. L. Evans and J. E. Cade \\ Nutritional Epidemiology Group, School of Food Science and Nutrition, University of Leeds, Leeds, UK
}

The trend of consuming large food portion sizes has occurred simultaneously with the rise in obesity prevalence, and therefore it is worth examining food portion size as a potential contributing risk factor for weight gain. However, limited studies have investigated this relationship particularly among adolescents. This study aims to investigate the association between food portion size for the top fifteen high energy dense-foods and body mass index (BMI), among British adolescents (aged 11-18 years), using the National Diet and Nutrition Survey (NDNS) 3 years combined from 2008 to $2011^{(1)}$.

Food portion sizes were estimated for all adolescents who had consumed the food at least once. Regression models with BMI as outcome were adjusted for age, sex and misreporting. Misreporting of energy intake (EI) was calculated based on Torun cut-off points ${ }^{(2)}$. There was a positive relationship between total EI in megajoule (MJ) and BMI (Coef. $=0.45,95 \%$ CI 0.24 to $0.66, P<0.001$ ) for the entire sample, even after stratifying it by misreporting. Larger portions of cream, biscuits, breakfast cereals, and buns, cakes $\&$ pastries were positively associated with higher BMI in adolescents, particularly among normal reporters. Biscuits were the highest contributors to adolescents daily EI with $4 \%$, followed by crisps $3.8 \%$, chocolate $3.6 \%$ and fat at $3.5 \%$. These findings highlight the need to increase adolescents' awareness regarding portion size and energy-dense foods in order to improve their food choice and future health outcomes.

\begin{tabular}{|c|c|c|c|c|c|c|c|c|c|}
\hline \multirow[b]{2}{*}{ Food group } & \multicolumn{3}{|c|}{ All adolescents } & \multicolumn{3}{|c|}{ Normal-reporter } & \multicolumn{3}{|c|}{ Under-reporter } \\
\hline & Coef.* & $95 \% \mathrm{CI}$ & $P$ value & Coef.* & $95 \% \mathrm{CI}$ & $P$ value & Coef.* & $95 \% \mathrm{CI}$ & $P$ value \\
\hline Fat & 0.02 & $-0.17,0.20$ & 0.86 & 0.01 & $-0.03,0.052$ & 0.63 & 0.016 & $-0.03,0.07$ & 0.5 \\
\hline Sweet spreads fillings and icing & -0.02 & $-0.07,0.030$ & 0.47 & -0.053 & $-0.13,0.024$ & 0.17 & -0.002 & $-0.07,0.064$ & 0.96 \\
\hline Crisps and savoury snacks & 0.012 & $-0.01,0.038$ & 0.35 & -0.001 & $-0.03,0.03$ & 0.98 & 0.015 & $-0.019,0.049$ & 0.38 \\
\hline Nuts and seeds & 0.01 & $-0.016,0.04$ & 0.44 & -0.011 & $-0.06,0.037$ & 0.65 & 0.016 & $-0.02,0.049$ & 0.32 \\
\hline Chocolate confectionery & 0.003 & $-0.01,0.013$ & 0.66 & 0.006 & $-0.01,0.021$ & 0.43 & 0.001 & $-0.014,0.02$ & 0.88 \\
\hline Biscuits (M\&R) & 0.002 & $-0.01,0.02$ & 0.5 & 0.028 & $0.001,0.06$ & 0.044 & 0.002 & $-0.017,0.020$ & 0.84 \\
\hline Buns cakes \& pastries $H$ & 0.008 & $-0.03,0.05$ & 0.68 & -0.017 & $-0.08,0.045$ & 0.562 & 0.012 & $-0.04,0.067$ & 0.67 \\
\hline Cheese (except cottage) & -0.01 & $-0.03,0.01$ & 0.4 & 0.026 & $-0.001,0.052$ & 0.053 & -0.02 & $-0.04,0.003$ & 0.08 \\
\hline Breakfast cereals Not $\mathrm{HF}$ & 0.004 & $-0.03,0.037$ & 0.82 & -0.001 & $-0.03,0.032$ & 0.95 & 0.007 & $-0.04,0.057$ & 0.77 \\
\hline Sugar & -0.001 & $-0.004,0.01$ & 0.56 & 0.025 & $-0.08,0.139$ & 0.66 & -0.034 & $-0.11,0.043$ & 0.38 \\
\hline Buns cakes \& pastries $M$ & -0.002 & $-0.015,0.01$ & 0.79 & 0.021 & $0.007,0.035$ & 0.005 & -0.013 & $-0.03,0.005$ & 0.16 \\
\hline Cream & 0.062 & $0.014,0.11$ & 0.013 & 0.07 & $0.002,0.148$ & 0.040 & 0.042 & $-0.029,0.114$ & 0.24 \\
\hline Meat pies and pastries $M$ & 0.000 & $-0.0,0.00$ & 0.75 & -0.01 & $-0.03,0.01$ & 0.21 & 0.009 & $-0.01,0.024$ & 0.23 \\
\hline Sugar confectionery & -0.013 & $-0.05,0.01$ & 0.42 & -0.001 & $-0.01,0.012$ & 0.99 & -0.012 & $-0.03,0.011$ & 0.29 \\
\hline Breakfast cereals $(\mathrm{HF})$ & 0.014 & $0.003,0.025$ & 0.013 & 0.007 & $-0.01,0.021$ & 0.33 & 0.017 & $0.002,0.030$ & 0.024 \\
\hline
\end{tabular}

(M: manufactured, H: homemade, HF: High Fibre) $* \mathrm{Kg} / \mathrm{m}^{2}$.

1. National Centre for Social Research, Medical Research Council. Resource Centre for Human Nutrition Research and University College London. Medical School, National Diet and Nutrition Survey, 2008-2011 [computer file].4th Edition. Colchester, Essex: UK Data Archive [distributor], January 2013. SN: 6533, http://dx.doi.org/10.5255/UKDA-SN-6533-3.

2. Torun B, Davies PS, Livingstone MB, Paolisso M, Sackett R, Spurr GB. (1996) Eur J of Clin Nutr. 50 (Suppl. 1): p. S37-S81. 